\title{
On the derivation of particle nucleation rates from experimental formation rates
}

\author{
A. Kürten ${ }^{1}$, C. Williamson ${ }^{1}$, J. Almeida ${ }^{2}$, J. Kirkby ${ }^{1,2}$, and J. Curtius ${ }^{1}$ \\ ${ }^{1}$ Institute for Atmospheric and Environmental Sciences, Goethe University of Frankfurt, 60438 Frankfurt am Main, Germany \\ ${ }^{2}$ CERN, 1211 Geneva, Switzerland \\ Correspondence to: A. Kürten (kuerten@iau.uni-frankfurt.de)
}

Received: 8 October 2014 - Published in Atmos. Chem. Phys. Discuss.: 29 October 2014

Revised: 30 March 2015 - Accepted: 31 March 2015 - Published: 17 April 2015

\begin{abstract}
Particle formation rates are usually measured at sizes larger than the critical size at which nucleation occurs. Due to loss of particles during their growth to the detection threshold, the measured formation rate is often substantially lower than the nucleation rate. For this reason a correction needs to be applied in order to determine the nucleation rate from the measured formation rate. Analytical formulae for the correction factor are provided in the literature. However, these methods were derived for atmospheric nucleation measurements and therefore need to be adjusted in order to be applied to chamber nucleation studies. Here we propose an alternative, numerical method that allows precise nucleation rates to be determined in arbitrary experimental environments. The method requires knowledge of the particle size distribution above detection threshold, the particle growth rate, and the particle loss rates as a function of particle size. The effect of self-coagulation, i.e., cluster-cluster collisions, is taken into account in the method.
\end{abstract}

\section{Introduction}

Aerosol nucleation, or new particle formation (NPF), is an important phenomenon taking place throughout the Earth's atmosphere (Kulmala et al., 2004). The key parameter of interest is the nucleation rate, which is defined as the formation rate $\left(\mathrm{cm}^{-3} \mathrm{~s}^{-1}\right)$ of new particles at the critical size. The critical size is the smallest size at which the growth rate of a particle is on average faster than its evaporation rate. This size depends mainly on the concentrations and other properties of the nucleating vapors, as well as on temperature. However, it is generally agreed that the critical size is somewhere below
$2 \mathrm{~nm}$ mobility diameter under atmospheric conditions (Kulmala et al., 2013). In fact it can be as small as two molecules in the case of barrierless, kinetically limited particle formation, where the dimer is already stable against evaporation (McMurry, 1980; Kürten et al., 2014).

Until recently the smallest mobility diameter that could be measured by a condensation particle counter (CPC) was 2.5 to $3 \mathrm{~nm}$ - which is substantially larger than the critical size. However, the detection limit of newly developed CPCs is as small as $1.2 \mathrm{~nm}$ in particle mobility diameter (Sgro and Fernández de la Mora, 2004; Iida et al., 2009; Vanhanen et al., 2011; Kuang et al., 2012a; Wimmer et al., 2013). Nevertheless, despite this progress the most widely used CPCs have detection thresholds at $2.5 \mathrm{~nm}$ or above. Moreover, care is needed when interpreting data from the newly developed CPCs since they can be sensitive to the chemical composition of the particles (Kangasluoma et al., 2014). Furthermore, CPC cutoff curves do not have the shape of a step function. Instead, detection of particles below the cutoff size (usually defined as the size $d_{50}$, where $50 \%$ of the particles are detected) is occurring to some extent and, if this includes clusters below the critical size, the accuracy of the derived nucleation rates can be strongly affected. For this reason, under certain conditions, it can still be more reliable to use a conventional CPC with a nominal cutoff around $3 \mathrm{~nm}$ for determining NPF rates. On the other hand, in order to minimize the corrections, it is advantageous to measure the formation rates as close as possible to the critical size.

Kerminen and Kulmala (2002) derived an analytical formula for correcting experimental particle formation rates to determine nucleation rates at a given critical size (abbreviated as the KK method in the following). This method was 
developed for atmospheric nucleation measurements, and a similar formula was also used by the McMurry group (Weber et al., 1997; McMurry et al., 2005). Several publications followed Kerminen and Kulmala (2002) to include additional effects, like a better description of the coagulation sink from particle size distribution measurements (Lehtinen et al., 2007), self-coagulation (Anttila et al., 2010), and a size-dependent growth rate (Korhonen et al., 2014). In addition to atmospheric measurements, nucleation studies in aerosol chambers or flow reactors have tremendously helped in the understanding of aerosol nucleation. Such experiments require an accurate method to derive the NPF rates. In this study the applicability of the previous methods to chamber experiments such as CLOUD (Cosmics Leaving OUtdoor Droplets) at CERN will be discussed (Kirkby et al., 2011; Almeida et al., 2013; Riccobono et al., 2014). Furthermore, we present here a new method that yields accurate results for any environment - be they chamber or atmospheric data provided the particle size distribution above a certain threshold size is known, as well as the particle growth rate, and where all loss processes are quantified as a function of size. The new method is verified with the results from a numeric aerosol model.

\section{Methods}

\subsection{Review of methods previously used for correcting the measured particle formation rate}

A lack of suitable instrumentation for the measurement of the particle number density at diameters below $\sim 3 \mathrm{~nm}$ required the application of a correction to derive the NPF rates close to the critical size (Weber et al., 1997; Kerminen and Kulmala, 2002). The corrections were derived for atmospheric particle measurements where the sink of the particles is usually dominated by the coagulation with larger pre-existing particles. In order to derive their analytical formulae, Kerminen and Kulmala (2002) as well as Lehtinen et al. (2007) made the following assumptions:

1. the only important sink for new particles is their coagulation with larger pre-existing particles,

2. the new particles grow at a constant rate,

3. the population of pre-existing particles remains unchanged during the new particle growth.

Finding an analytical expression for the relationship between the nucleation rate at a smaller size $\left(d_{p 1}\right)$ and a larger size $\left(d_{p 2}\right)$ requires taking into account the size dependency of the coagulation coefficient. However, the coagulation coefficient does not follow an expression, which can be analytically integrated (Seinfeld and Pandis, 2006). Therefore, KK made the assumption that the coagulation coefficient decreases with particle size $d_{p}$ to the second power, i.e.,

$K\left(d_{p}, d_{j}\right) \cdot d_{p}^{2}=K\left(d_{p 1}, d_{j}\right) \cdot d_{p 1}^{2}$,

where $K$ is the coagulation coefficient and $d_{j}$ is the diameter of pre-existing particles. This assumption leads to the following analytical expression, which connects the particle formation rates $J$ at different sizes:

$$
\begin{aligned}
& J\left(d_{p 1}\right)= \\
& \quad J\left(d_{p 2}\right) \cdot \exp \left(\frac{\mathrm{CS}\left(d_{p 1}\right)}{\mathrm{GR}} \cdot d_{p 1}^{2} \cdot\left(\frac{1}{d_{p 1}}-\frac{1}{d_{p 2}}\right)\right),
\end{aligned}
$$

where

$$
\mathrm{CS}\left(d_{p 1}\right)=\sum_{j} K\left(d_{p 1}, d_{j}\right) \cdot N_{j}
$$

is the coagulation sink for the nucleated particles due to larger pre-existing particles $N_{j}$ and GR is the particle growth rate (typically expressed in $\mathrm{nm} \mathrm{h}^{-1}$ ). However, depending on the ambient particle size spectrum, the power dependency from Eq. (1) can be weaker; for example, Fig. 1 shows the calculated coagulation coefficient between nanometer-sized particles and particles of $100 \mathrm{~nm}$ in diameter (solid black line, upper panel). The power dependency follows a value of -1.5 rather than -2 (see Eq. 1), and for smaller particles the magnitude of the slope becomes even smaller (colored lines in Fig. 1, upper panel). The indicated slopes are reported for the size range between $d_{p 1}=1.7 \mathrm{~nm}$ and $d_{p 2}=3.2 \mathrm{~nm}$ (mobility diameters) because these are used in the CLOUD experiment (Kirkby et al., 2011; Almeida et al., 2013; Riccobono et al., 2014) and also in the later sections of this text. Note that the mobility diameter can be obtained by adding a constant value of $0.3 \mathrm{~nm}$ to the geometric diameter ( $\mathrm{Ku}$ and Fernandez de la Mora, 2009).

Realizing that the power dependency from Eq. (1) depends on the conditions during a nucleation event, Lehtinen et al. (2007), in a follow-up publication, dealt with introducing the real power dependency derived from atmospheric size distribution measurements. This led to the following formulation for the size correction:

$J\left(d_{p 1}\right)=J\left(d_{p 2}\right) \cdot \exp \left(\gamma \cdot d_{p 1} \cdot \frac{\mathrm{CS}\left(d_{p 1}\right)}{\mathrm{GR}}\right)$,

with

$\gamma=\frac{1}{s+1} \cdot\left[\left(\frac{d_{p 2}}{d_{p 1}}\right)^{s+1}-1\right]$,

where the parameter $s$ is the slope of the coagulation coefficient with particle size.

Furthermore, recent findings from atmospheric growth rate measurements indicate that the GR can be a function of particle size (Kuang et al., 2012b; Kulmala et al., 2013). 

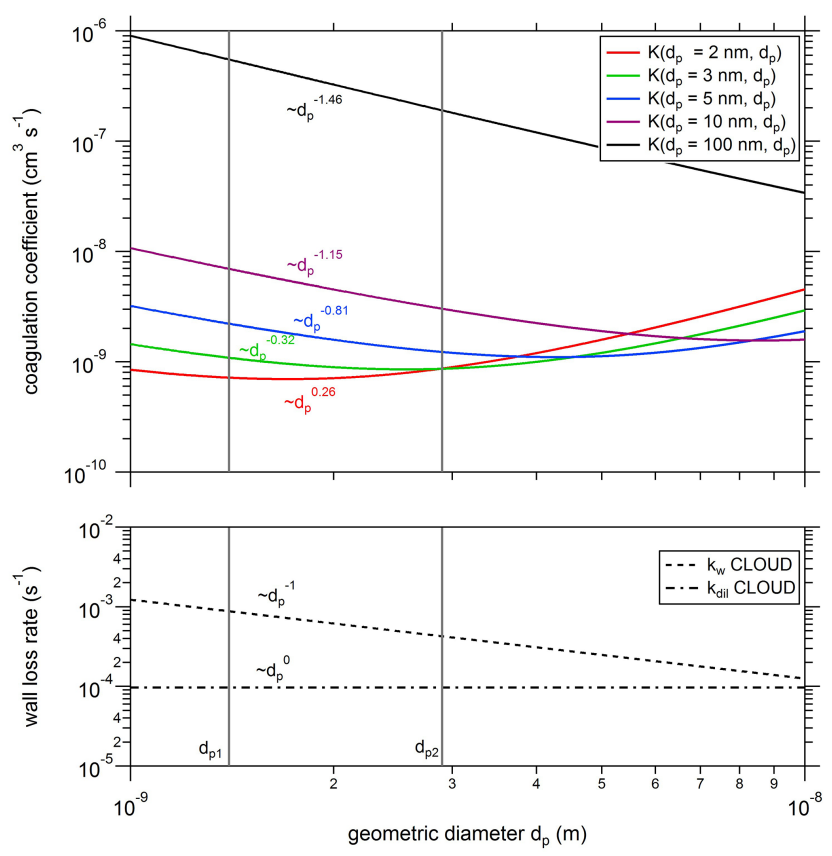

Figure 1. Coagulation coefficient, $K$, as a function of particle size, $d_{p}$ (upper panel). Coagulation coefficients are calculated between two particles, where one particle has a constant size (indicated in the legend of the figure) and the second particle diameter varies between 1 and $10 \mathrm{~nm}$. The wall loss rate for the CLOUD chamber as a function of particle size is shown by the dashed curve (lower panel), whereas the dilution rate is indicated by the dash-dotted line. Slopes of the curves are indicated for the range between $d_{p 1}(1.4 \mathrm{~nm}$, i.e., $1.7 \mathrm{~nm}$ in mobility diameter) and $d_{p 2}(2.9 \mathrm{~nm}$, i.e., $3.2 \mathrm{~nm}$ in mobility diameter).

Therefore, Korhonen et al. (2014) extended the analytical solution from Eqs. (4) and (5) and included the effect of a sizedependent GR, which can either vary linearly with particle size or according to a power-law dependency. Another effect that can become important when the population of particles between $d_{p 1}$ and $d_{p 2}$ becomes large is self-coagulation. This effect was considered recently by Anttila et al. (2010). While we will also deal with the effects of a size-dependent GR (Sect. 3.1) and self-coagulation (Sect. 3.3), we will first focus on the question of how far atmospheric nucleation and nucleation within a chamber experiment are comparable in terms of their loss processes in the next section.

\subsection{Relevant losses in chamber experiments}

The dominant particle loss mechanism for seedless chamber nucleation experiments is generally due to collisions with the walls of the vessel and possibly also due to dilution of the chamber gas. Large $(3 \mathrm{~m})$ chambers such as CLOUD have wall loss rates (around $0.001 \mathrm{~s}^{-1}$ at $1 \mathrm{~nm}$ ) similar to the loss rates onto pre-existing aerosols in a pristine atmospheric environment. We will address here to what extent these two environments are equivalent.
The wall loss rate in chamber experiments can be expressed by (Crump and Seinfeld, 1981; Metzger et al., 2010)

$k_{w}\left(d_{p}\right)=C \cdot \sqrt{D\left(d_{p}\right)}$,

where $D\left(d_{p}\right)$ is the diffusivity of a particle with size $d_{p}$ and $C$ is an empirical factor that depends on the chamber dimensions and turbulent mixing. The diffusivity of a particle can be calculated from the Stokes-Einstein relationship according to (Hinds, 1999)

$D\left(d_{p}\right)=\frac{k_{\mathrm{B}} \cdot T \cdot C_{\mathrm{C}}}{3 \cdot \pi \cdot \eta \cdot d_{p}}$,

which depends on the Boltzmann constant $k_{\mathrm{B}}$, the temperature $T$, gas viscosity $\eta$, and the Cunningham correction factor $C_{\mathrm{C}}$. The latter is a function of the gas mean free path and the particle diameter. At small particle sizes the Cunningham correction factor is approximately proportional to $d_{p}^{-1}$, and so the wall loss rate can be approximated by

$k_{w}\left(d_{p}\right)=\frac{C^{\prime}}{d_{p}}$,

where $C^{\prime}$ is an empirical constant determined from a leastsquares fit by taking into account measured wall loss rates of sulfuric acid monomers and particles in different size bins. Figure 1 shows the wall loss rate for the CLOUD chamber as a function of $d_{p}$ (dashed curve, lower panel), where the value of $C^{\prime}$ is approximately $0.001 \mathrm{~nm} \mathrm{~s}^{-1}$. The wall loss rate decreases by $\sim d_{p}^{-1}$, which is much weaker than the originally assumed power dependency of $\sim d_{p}^{-2}$ for loss to atmospheric particles (Eq. 1).

In addition to wall loss, another mechanism which affects the particle number density in a chamber experiment is dilution of the chamber gas. Instruments can take considerable amounts of the chamber gas, and this gas needs to be replenished in order to maintain a constant pressure. The CLOUD chamber has a volume of $26.1 \mathrm{~m}^{3}$, while the instruments typically use $150 \mathrm{~L} \mathrm{~min}^{-1}$. This leads to a dilution rate of $k_{\text {dil }}=9.6 \times 10^{-5} \mathrm{~s}^{-1}$, which is independent of particle size (see dash-dotted black line in the lower panel of Fig. 1).

If coagulation with larger pre-existing aerosols is neglected, which is well justified in a seedless chamber experiment, the two main loss mechanisms - wall loss and dilution - can be used to derive an analytical solution for the NPF rate at a small size. This is achieved by replacing the coagulation loss term in Eq. (4) from Lehtinen et al. (2007) with $k_{w}\left(d_{p}\right)$ 
and $k_{\mathrm{dil}}$ :

$$
\begin{aligned}
\frac{\mathrm{d} J\left(d_{p}\right)}{\mathrm{d} d_{p}} & =-\frac{\text { loss rate }}{\mathrm{GR}} \cdot J\left(d_{p}\right) \\
& =-\frac{1}{\mathrm{GR}} \cdot\left(\frac{C^{\prime}}{d_{p}}+k_{\mathrm{dil}}\right) \cdot J\left(d_{p}\right) .
\end{aligned}
$$

In this case, integration yields

$$
\begin{aligned}
& J\left(d_{p 1}\right)=J\left(d_{p 2}\right) \\
& \quad \cdot \exp \left(\frac{1}{\mathrm{GR}} \cdot\left(C^{\prime} \cdot \ln \left(\frac{d_{p 2}}{d_{p 1}}\right)+k_{\mathrm{dil}} \cdot\left(d_{p 2}-d_{p 1}\right)\right)\right) .
\end{aligned}
$$

The identical result would follow from Eqs. (4) and (5) by taking the limit for $s \rightarrow-1$ to take into account wall loss, and by taking $s=0$ for loss due to dilution.

In conclusion, the KK method and also the follow-up versions should only be applied to chamber nucleation experiments after applying the necessary adjustments. Equation (10) provides a useful analytical formula for conditions in which coagulation can be neglected. The data from Fig. 1 provide a guide as to the relative importance of the different loss mechanisms for the CLOUD chamber. The wall loss rate for the relevant sizes between 1.4 and $2.9 \mathrm{~nm}$ is on the order of $10^{-3} \mathrm{~s}^{-1}$. Depending on particle size the coagulation coefficient is in the range $10^{-9}$ to $10^{-8} \mathrm{~cm}^{-3} \mathrm{~s}^{-1}$, which indicates that particle number densities between $10^{5}$ and $10^{6} \mathrm{~cm}^{-3}$ are required in order to reach similar effects for coagulation and wall loss. At this point it is also worth mentioning that all the expressions derived so far are based on the assumption that nucleation and particle growth is driven by the condensation of monomers (Lehtinen et al., 2007) and that cluster-cluster collisions are unimportant. The effect of cluster-cluster collisions will be discussed in Sect. 2.4.

The important conclusion that follows from the comparison of Eqs. (2), (4), and (10) is that experiments and atmospheric environments with similar sink rates cannot be directly compared before corrections are applied, because not only the magnitude of the sink is important but also the dependency of the loss rate as a function of particle size. Despite the practicability of Eq. (10), a new method is required which additionally takes into account coagulation as well as self-coagulation.

\subsection{New method to derive the nucleation rate from the experimental formation rate}

We will assume that the size distribution above a certain threshold size $\left(d_{p 2}\right)$ is known, and furthermore that the size between two adjacent bins differs by one molecule only. For the following discussion it is useful to add $m$ to all bin indices, although the original size distribution contains $n$ size bins ranging from 1 to $n$. In this case the size $d_{p 2}$ corresponds to the bin with the index $m+1$ (Fig. 2). The formation rate of

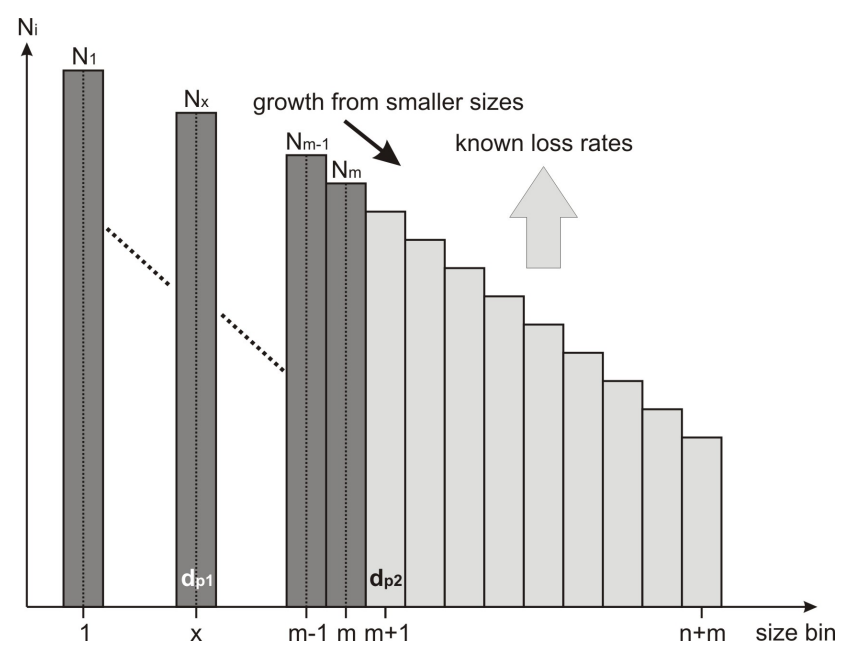

Figure 2. The original size distribution above the cutoff size $d_{p 2}$ (size bin $m+1$ ) is shown in light grey. The loss rate of particles and the rate of change of the particle concentration in this size range must be compensated for by the formation rate due to smaller particles growing into the measured size range. This knowledge can be incrementally extended to bins at smaller sizes in a stepwise process, finally reaching the smaller size, $d_{p 1}($ size bin $x$ ).

particles at and above $d_{p 2}$ can then be calculated from

$$
\begin{aligned}
J_{\geq m+1} & =\frac{\mathrm{d} N_{\geq m+1}}{\mathrm{~d} t}+\sum_{i=m+1}^{n+m}\left(k_{w, i} \cdot N_{i}\right)+k_{\mathrm{dil}} \cdot N_{\geq m+1} \\
& +\sum_{i=m+1}^{n+m}\left(\sum_{j=i}^{n+m} \delta_{i, j} \cdot K_{i, j} \cdot N_{j} \cdot N_{i}\right),
\end{aligned}
$$

where double counting of collisions between particles in the same size bin is avoided by the factor (Seinfeld and Pandis, 2006)

$\delta_{i, j}=\left\{\begin{array}{ll}0.5 & \text { if } i=j \\ 1 & \text { if } i \neq j\end{array}\right.$.

The first term on the right-hand side takes into account nonsteady-state conditions by means of the time derivative of the total particle number density (sum of the particle concentrations from bin $m+1$ to $n+m$ ). The remaining three terms on the right-hand side describe the loss processes of neutral particles in a chamber experiment: wall loss, loss due to dilution of the chamber gas (independent of particle size), and coagulation loss between particles of all size bins. Note that the index $i$ runs from $m+1$ to $n+m$ and the index $j$ from $i$ to $n+m$. In this way, the collisions between the bins $i$ and $j$ are not counted twice. Since we are looking at formation rates larger than a certain size, collision products will remain in the size range under consideration and therefore loss due to coagulation between bins $i$ and $j$ only has to be taken into account once. The formation rate at $d_{p 2}$ can also be calcu- 
lated from

$$
\begin{aligned}
J_{\geq m+1} & =J_{\geq m+2}+\frac{\mathrm{d} N_{m+1}}{\mathrm{~d} t} \\
& +\left(k_{w, m+1}+k_{\mathrm{dil}}+\sum_{j=m+1}^{n+m} \delta_{m+1, j} \cdot K_{m+1, j} \cdot N_{j}\right) \\
& \cdot N_{m+1} .
\end{aligned}
$$

This equation allows for calculation of the formation rate at a smaller size from the NPF rate at the next larger size. Here the time derivative of $N_{m+1}$ refers to the concentration of particles in the size bin $m+1$, whereas in Eq. (11) it refers to all particles at and above index $m+1$.

In order to calculate the formation rate $d_{p 1}$ let us now introduce $x+1$ new size bins, which extends the size distribution towards the smaller sizes (Fig. 2). Kerminen et al. (2004) also introduced extra size bins in order to increase the accuracy of their analytical formula, which connects the nucleation rate and a formation rate at a larger size similar to Eq. (2). Extending the size distribution towards smaller sizes requires calculation of the number concentration $N_{m}$ in the first new bin. However, Eq. (13) does not allow this directly; therefore additional information is required. This information can be taken from the growth rate of the particles. The formation rate and the growth rate (GR) are connected by the following equation (Heisler and Friedlander, 1977; Lehtinen et al., 2007):

$$
J_{m+1}=\mathrm{GR}_{m} \cdot \frac{N_{m}}{d_{p, m+1}-d_{p, m}} .
$$

This relationship was used to describe the flux of particles due to collisions with monomers. In such a case particles can grow only from one size bin to the next larger bin without "jumping" into an even larger bin due to cluster-cluster collisions. However, for the moment we will assume that Eq. (14) is also valid for the case where cluster-cluster collisions are relevant if appropriate definitions for the growth rate and NPF rate are being used, and we will justify this assumption later in Sect. 2.4. Using of the formation rate and growth rate relationship, the particle number concentration can be calculated for the first new size bin $\left(N_{m}\right)$ from the following relationship:

$$
\begin{aligned}
N_{m} & =\frac{d_{p, m+1}-d_{p, m}}{\mathrm{GR}_{m}} \cdot\left(J_{\geq m+2}+\frac{\mathrm{d} N_{m+1}}{\mathrm{~d} t}+\left(k_{w, m+1}\right.\right. \\
& \left.\left.+k_{\mathrm{dil}}+\sum_{j=m+1}^{n+m} \delta_{m+1, j} \cdot K_{m+1, j} \cdot N_{j}\right) \cdot N_{m+1}\right) .
\end{aligned}
$$

In the limiting case where particle formation and growth is dominated by the addition of monomers, this method is accurate at steady state provided that knowledge about the growth rate is available initially.

When applying the method, the particle growth rate $\mathrm{GR}_{m}$ is required for calculating the first unknown concentration.
Strictly, the growth rate is not known at the index $m$ (because the known size distribution starts at index $m+1$ by definition; see Fig. 2) but can only be calculated at the next larger index using Eq. (14) by adjusting all indices to the next larger bin. According to Eq. (15), the GR would need to be updated in every reconstruction step. Nevertheless, we have found from numerical simulation (see later sections) that the method is numerically more stable if a constant GR at index $m+1$ is used for all iterative steps. However, if accurate knowledge about a size-dependent GR is available, it can be easily implemented in the method.

In Eq. (15) all quantities are known except the value of $N_{m}$ (if $\mathrm{GR}_{m}$ is approximated by $\mathrm{GR}_{m+1}$ ). Once $N_{m}$ is found, the formation rate $J_{m}$ can be calculated and the process repeated with the next smaller size bin (index $m-1$ ). In this way the complete particle spectrum above $d_{p 1}$ (containing now $n+x+1$ size bins) can be recreated until the final formation rate $J_{d_{p 1}}$ (at index $x$ ) is calculated. The underlying assumption is that growth above this size is purely kinetic (no evaporation), which is likely a good assumption for most chemical systems and the atmospheric data (e.g., Chen et al., 2012). A similar approximation was made by Nieminen et al. (2010) when deriving an analytical formula for calculating growth rates where the vapor pressure of the condensing species has been set to zero. However, in future studies, one could examine the effect of evaporation at sizes larger than the critical diameter on the method and attempt to implement it in a similar fashion as Olenius et al. (2014) in their study about the effect of monomer collisions on the growth rates.

In order to test the relative importance of self-coagulation on the magnitude of the formation rate correction it is also possible to take into account only particles at and above $m+1$ in all reconstruction steps in the last term on the right-hand side of Eq. (15). In Sect. 3.3 we will discuss under which circumstances this can be done without sacrificing too much accuracy.

\subsection{Relationship between particle formation rate and growth rate including cluster-cluster collisions}

In a recent publication, Olenius et al. (2014) investigated the relationship between $J$ and GR as well as different methods for deriving the GR due to monomer collisions. The method introduced here should also be applied to conditions where new particle formation is proceeding at the kinetic limit, i.e., where all cluster evaporation rates are zero. Under such conditions the cluster concentrations are quite high in comparison to the monomer concentration, e.g., the dimer concentration can be $\sim 20 \%$ of the monomer concentration (McMurry, 1980; Chen et al., 2012; Kürten et al., 2014). In this case, the particle formation as well as the particle growth cannot be described by monomer collisions only and cluster-cluster collisions have to be taken into account. Therefore, Eq. (14) might not be valid anymore. In the following we will investigate whether the relationship from Eq. (14) can still be used. 
Following a similar approach as Olenius et al. (2014) but taking into account cluster collisions and neglecting the effect of evaporation, the particle growth rate for particles in the size bin $m$ can be defined as

$$
\begin{aligned}
\mathrm{GR}_{m} & =\frac{\mathrm{d} d_{p, m}}{\mathrm{~d} t}=\sum_{i=1}^{m} \delta_{i, j} \\
& \cdot\left(\left(d_{p, m}^{3}+d_{p, i}^{3}\right)^{1 / 3}-d_{p, m}\right) \cdot K_{i, m} \cdot N_{i} .
\end{aligned}
$$

Equation (16) indicates that the clusters in the size bin $m$ can grow by collisions with all smaller clusters. We will assume that a particle in size bin $m$ contains $m$ monomers with a mass $u$ and density $\rho$ :

$d_{p, m}=\left(\frac{6 \cdot m \cdot u}{\pi \cdot \rho}\right)^{1 / 3}=d_{p, \text { mono }} \cdot m^{1 / 3}$,

where $d_{p, \text { mono }}$ is the diameter of the monomer. Multiplication of the growth rate from Eq. (16) by $N_{m} /\left(d_{p, m+1}-d_{p, m}\right)$ and using the Taylor expansion

$$
\begin{aligned}
\left((m+i)^{1 / 3}-m^{1 / 3}\right) & =m^{1 / 3} \cdot\left(\left(1+\frac{i}{m}\right)^{1 / 3}-1\right) \\
& \approx \frac{i}{3 \cdot m^{2 / 3}}
\end{aligned}
$$

leads to the following expression:

$\mathrm{GR}_{m} \cdot \frac{N_{m}}{d_{p, m+1}-d_{p, m}} \approx \sum_{i=1}^{m} \delta_{i, j} \cdot i \cdot K_{i, m} \cdot N_{i} \cdot N_{m}$.

On the other hand, the particle formation rate $J_{\geq m+1}$ can be defined as

$J_{\geq m+1}=0.5 \cdot \sum_{i+j \geq m+1} \delta_{i, j} \cdot K_{i, j} \cdot N_{i} \cdot N_{j}$,

where $i$ and $j$ have to be smaller than $m+1$. From this definition it follows that Eq. (19) cannot be cast into the form of Eq. (20) because the equation involving the growth rate only considers collisions where one collision partner always belongs to the size bin $m$. Instead, Eq. (20) involves collisions where none of the collision partners is fixed to one size bin in the summation. Therefore, we were not able to find an analytical solution in terms of bringing Eqs. (19) and (20) into agreement. However, we can argue qualitatively that the two equations are yielding approximately the same results for certain conditions.

The accurate definition of $J_{\geq m+1}$ (Eq. 20) is visualized schematically in Fig. 3a, whereas Eq. (19) is indicated in Fig. 3b. For the monomer there is only one possibility for contributing to $J_{\geq m+1}$ in both cases. However, the dimer can contribute to $J_{\geq m+1}$ due to collisions with particles in bin $m$ and bin $m-1$. The approximation (Eq. 19) only takes into account collisions between dimers and particles in bin a)

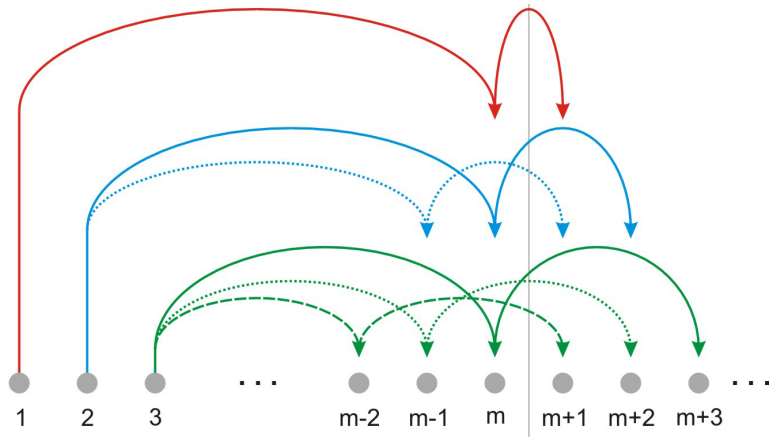

b)

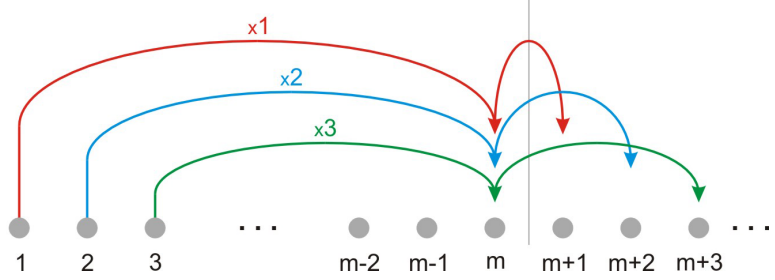

Figure 3. (a) Particle formation rate $J_{\geq m+1}$ due to collisions of monomers and clusters. (b) Approximation of the particle formation rate including the growth rate definition according to Eq. (19). See text for details.

$m$. However, the dimer collision is taken twice and the second collision can therefore compensate for the collision between dimers and particles in bin $m-1$ from Eq. (20). For the trimer the situation is similar; in the accurate case the trimer has three possibilities (i.e., three different collisions) to contribute to $J_{\geq m+1}$. The approximation (Eq. 19) is taking into account only one collision, but it is multiplied by a factor of 3. This mechanism is the same for the collisions involving larger clusters. Therefore, we can conclude that

$\mathrm{GR}_{m} \cdot \frac{N_{m}}{d_{p, m+1}-d_{p, m}} \approx J_{\geq m+1}$

applies also for conditions where cluster-cluster collisions become important (note the " $\geq$ " sign on the right-hand side). The requirement is that the cluster concentrations do not change strongly in the region around bin $m$ and that the contribution of clusters to new particle formation and growth becomes negligible at some index smaller than $m$. Under what circumstances the relationship from Eq. (21) is valid needs to be studied in more detail in the future. However, the benefit of the method from Sect. 2.3 is that even if the relationship from Eq. (21) introduces inaccuracies, these are very likely small because its effects should cancel out. $\mathrm{GR}_{m+1}$ for Eq. (15) is calculated from the relationship in Eq. (21), and the same relationship is used to calculate $N_{m}$ in Eq. (15). Therefore, we expect the error due to this approximation to be small, and the numerical simulations shown in the following sections support this assumption. 


\subsection{Kinetic model for testing the universal method}

A numerical model was developed recently for the CLOUD chamber to simulate the formation and growth of uncharged sulfuric acid-dimethylamine particles (Kürten et al., 2014). The model assumes that particles grow from monomers by condensation and coagulation. Due to the arguments presented by Kürten et al. (2014), it has been concluded that $\mathrm{H}_{2} \mathrm{SO}_{4} \cdot\left(\left(\mathrm{CH}_{3}\right)_{2} \mathrm{NH}\right.$ clusters (abbreviated as $\mathrm{SA} \cdot \mathrm{DMA}$ ) constitute the basic "monomer" for the formation of particles in a system of sulfuric acid (SA) and dimethylamine (DMA). Assuming unit sticking efficiency and zero evaporation rate, good agreement is found between the model and the experimentally measured neutral clusters.

The kinetic model is based on McMurry (1980). The timedependent balance equation for the monomer concentration $N_{1}$ is

$$
\frac{\mathrm{d} N_{1}}{\mathrm{~d} t}=P_{1}-\left(k_{1, w}+k_{\mathrm{dil}}+\sum_{j=1}^{N} K_{1, j} \cdot N_{j}\right) \cdot N_{1}
$$

and, for all larger clusters $(k \geq 2)$,

$$
\begin{aligned}
\frac{\mathrm{d} N_{k}}{\mathrm{~d} t} & =\frac{1}{2} \cdot \sum_{i+j=k} K_{i, j} \cdot N_{i} \cdot N_{j} \\
& -\left(k_{w, k}+k_{\mathrm{dil}}+\sum_{j=1}^{N} K_{k, j} \cdot N_{j}\right) \cdot N_{k} .
\end{aligned}
$$

Here, $P_{1}$ is the production rate of the monomers, $k_{w}$ the wall loss rate, $k_{\text {dil }}$ the dilution rate, and $K$ the coagulation coefficient.

The original model calculated concentrations of clusters ranging from dimer up to clusters of several thousand molecules. Each size bin was represented by a single cluster with a fixed number of molecules (or SA - DMA clusters, which are each treated as one molecule). The maximum particle size that can be reached with reasonable computation time is a few nanometers, which is too small for the current study. Therefore we incremented the size by one molecule for the first 100 bins (linear bins) and by a constant geometrical factor for the next 100 bins (geometric bins; see, for example, Landgrebe and Pratsinis, 1990, or Lovejoy et al., 2004). With this method, a size of $\sim 30 \mathrm{~nm}$ can be reached using a geometrical factor of 1.023, which is suitable for the present study. The sizes of $d_{p 1}$ and $d_{p 2}$ do, however, fall into the size range of the linear bins.

In addition to the kinetic modeling, we have also introduced evaporation rates for the dimer and the trimer (evaporation rates not included in Eqs. 22 and 23 for simplicity). These simulations are used to investigate situations where nucleation and particle growth is dominated by the addition of monomers, because if the evaporation rates for the smallest clusters are sufficiently high, their concentrations become very small and will therefore not contribute significantly to NPF and growth. Although not directly relevant for the sulfuric acid-dimethylamine system, we have calculated the dimer and trimer evaporation rates at $223.15,248.15$, and $278.15 \mathrm{~K}$ at $38 \%$ RH from the data presented by Hanson and Lovejoy (2006). Their thermodynamic data were derived for the binary system of sulfuric acid and water. However, the calculated formation rates are not meant to be representative of binary nucleation; rather, they only serve to demonstrate the effect of going from purely kinetic nucleation to nucleation with a relatively large barrier $(278 \mathrm{~K}$ data). Kinetic nucleation will include collisions with monomers and also show a significant effect from clusters, whereas the new particle formation at $278 \mathrm{~K}$ will be dominated by monomer collisions. The other two temperatures show the transition from purely kinetic nucleation to nucleation dominated by monomer additions.

Particle formation rates that have been calculated from the model serve as the reference formation rates to which the reconstructed formation rates can be compared to. We have implemented two separate procedures to calculate the NPF rates, where the first one is following the approach based on Eq. (11) by taking into account all loss processes, while the second one follows the production of particles from two smaller clusters (Eq. 20). The two methods yield exactly the same result, which is a good verification of the kinetic model in this respect.

\section{Discussion}

Figure 4 shows the result of the kinetic model simulation for a monomer (molecular weight of $143 \mathrm{~g} \mathrm{~mol}^{-1}$ and density of $1.47 \mathrm{~g} \mathrm{~cm}^{-3}$ ) production rate of $8.8 \times 10^{4} \mathrm{~cm}^{-3} \mathrm{~s}^{-1}$, after $1.5 \times 10^{4} \mathrm{~s}$. Integration of Eqs. (22) and (23) yields the displayed size distribution (grey bars). Although the particles continue to grow, the populations at smaller sizes (below about $10 \mathrm{~nm}$ ) are close to steady state. Since the total particle number concentration is dominated by these smaller particles, time dependency can be neglected in the following, but will be revisited in Sect. 3.2. The size distribution (grey bars in Fig. 4) is obtained after normalizing the concentrations by the number of molecules per bin.

The new universal method to derive a particle formation rate at a smaller size $d_{p 1}$ has been applied to the data shown in Fig. 4. A threshold size $d_{p 2}=3.2 \mathrm{~nm}$ (corresponding to $2.9 \mathrm{~nm}$ geometric diameter) has been chosen. Starting with the size distribution for particles equal to or larger than $2.9 \mathrm{~nm}, 71 \mathrm{new}$ bins were introduced to reach the size $d_{p 1}$ at $1.7 \mathrm{~nm}(1.4 \mathrm{~nm}$ geometric diameter). The red line shows the recreated size distribution obtained by this method. A constant growth rate of $3.81 \mathrm{~nm} \mathrm{~h}^{-1}$ was chosen, corresponding to the value given by a numeric model calculation for a particle in the size bin $m+1$. As can be seen, the reconstruction works well for the first few size bins and then starts to deviate somewhat from the correct values. This occurs since the GR 


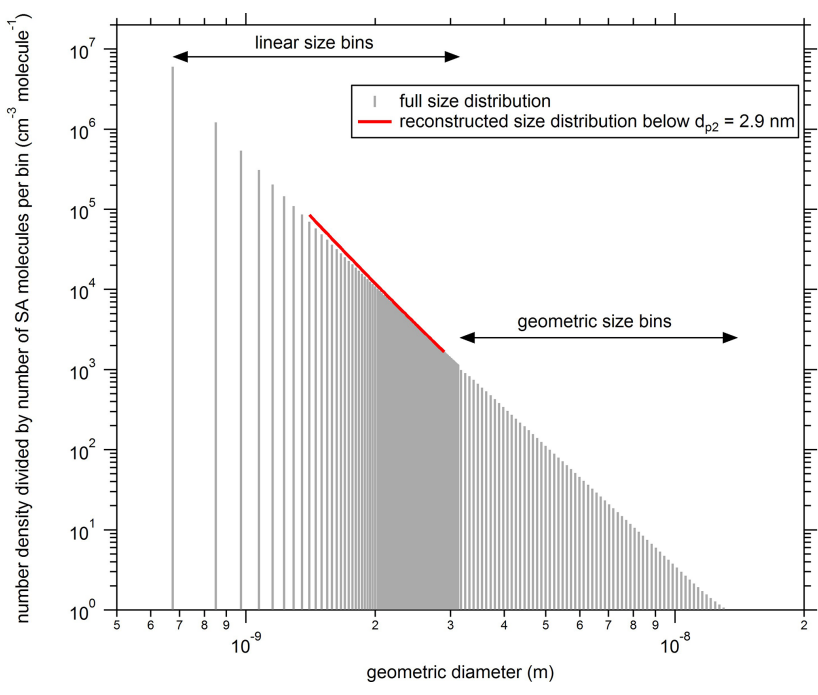

Figure 4. Modeled and reconstructed particle size distribution for kinetic nucleation. The model uses different definitions for the first 100 size bins (up to $\sim 3.1 \mathrm{~nm}$ ) and the last 100 size bins $(>\sim 3.1 \mathrm{~nm})$. In the first 100 size bins, the number of molecules in the particles increases by one between each bin, whereas in the next 100 bins the particle diameter is increased by a constant factor between each bin. Normalizing the concentration by the number of molecules per bin leads to the shown size distribution (grey bars). The reconstructed size distribution using the new method described here is shown by the solid red line, starting from the particle distribution above $2.9 \mathrm{~nm}$.

is not exactly constant with size, and slightly increases when approaching $d_{p 1}$ (see Sect. 3.1).

\subsection{Size-dependent growth rate}

The growth rate which is used for the reconstruction is calculated from

$\mathrm{GR}_{m+1}=J_{\geq m+2} \cdot \frac{d_{p, m+2}-d_{p, m+1}}{N_{m+1}}$.

Our studies with the kinetic model indicate that GR is only weakly dependent on particle size in the range between critical size and detection threshold. In the example shown in Fig. 4 there is less than $20 \%$ variation. However, the model does not include the effects of evaporation or of a spectrum of condensable vapors with different volatilities. Therefore care has to be taken when applying size corrections to atmospheric particle formation rates. The GR should ideally be measured over a wide range of diameters (Kulmala et al., 2013). In this case analytical solutions for the KK method can be found for certain size-dependent GRs (Korhonen et al., 2014). These considerations underscore the importance of directly measuring the particle GR in the sub- $3 \mathrm{~nm}$ size range, as well as at larger sizes. With this information the effect of particle evaporation can be separated from the uncertainties due to size-dependent particle GR. In the absence

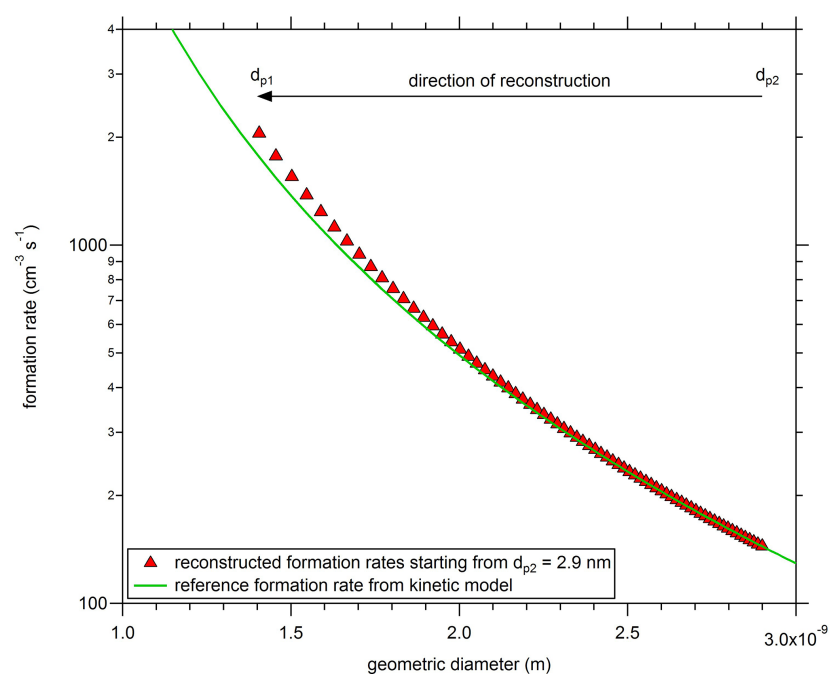

Figure 5. Formation rates as a function of particle size for kinetic nucleation. Formation rates simulated with the kinetic model are shown by the green line. Reconstructed particle formation rates starting at $d_{p 2}=2.9 \mathrm{~nm}$ and ending at $d_{p 1}=1.4 \mathrm{~nm}$ using a constant GR (taken at $d_{p 2}$ ) are shown by the red triangles.

of such measurements, a detailed error analysis is required to bracket the range of GR uncertainty and its impact on the derived nucleation rates.

A comparison between the accurate solution for the NPF rates and the ones from the reconstruction method as a function of particle size is shown in Fig. 5. The accurate solution from the kinetic model is shown by the solid green line, while the results from the reconstruction method are indicated by the red triangles. Due to the slight size dependency of the growth rate (it increases slightly with decreasing size), the reconstructed NPF rates are somewhat higher than the accurately calculated values. The maximum deviation occurs at the smallest size and reaches $\sim 17 \%$ in this example. Given the fact that the formation rate $J\left(d_{p 1}\right)$ is more than a factor of 12 higher than $J\left(d_{p 2}\right)$, this is a rather small deviation.

\subsection{Time evolution in a simulated chamber nucleation experiment}

Using a kinetic model simulation, we show in Fig. 6 an example of the time-dependent formation rates $J$ for the particle sizes $d_{p 1}(1.4 \mathrm{~nm}$ geometric diameter; solid green line) and $d_{p 2}(2.9 \mathrm{~nm}$ geometric diameter; solid blue line). In addition, the rate of change of particle concentration $\mathrm{d} N / \mathrm{d} t$ (dashed lines) above the size thresholds $d_{p 1}$ and $d_{p 2}$ are shown. The formation rates $J$ are directly obtained from the model using Eq. (11) and the size distribution. Interestingly, the formation rates overshoot before they reach an almost constant value. This overshoot is explained by the absence of larger particles at the beginning of the experiment. Therefore the loss rate is smaller at the beginning, which allows for faster forma- 


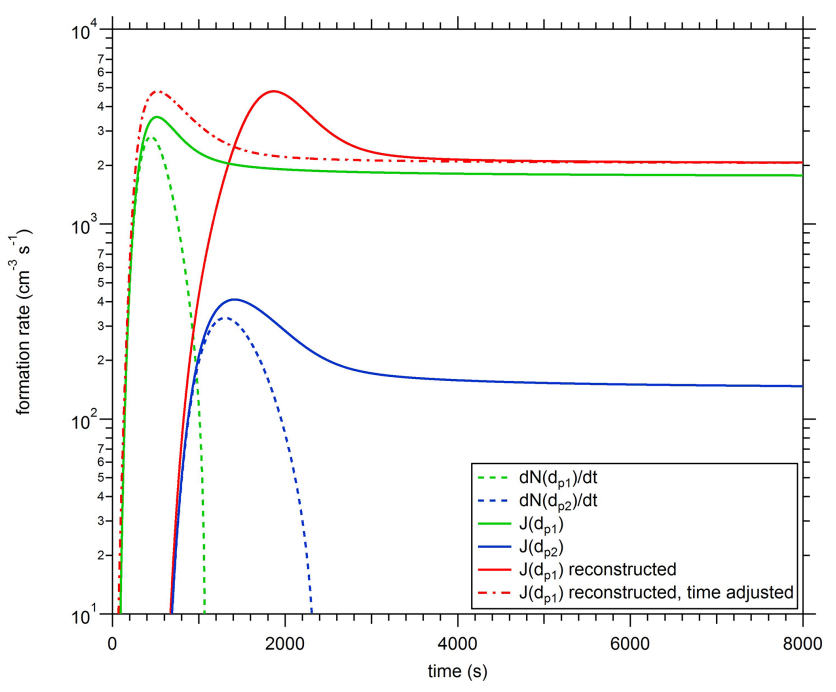

Figure 6. Particle formation rates $J\left(\mathrm{~cm}^{-3} \mathrm{~s}^{-1}\right.$, solid lines) and change in particle concentration $\mathrm{d} N / \mathrm{d} t\left(\mathrm{~cm}^{-3} \mathrm{~s}^{-1}\right.$, dashed lines) shown for two different sizes, $d_{p 1}=1.4 \mathrm{~nm}$ (green lines) and $d_{p 2}=2.9 \mathrm{~nm}$ (blue lines). The data are from a kinetic model calculation. The reconstructed $J\left(d_{p 1}\right)$ is shown by the solid red line. Through use of a time correction, the reconstructed $J\left(d_{p 1}\right)$ are shifted to earlier times (dash-dotted red line).

tion rates. Once the larger particles start to form, the loss rate increases until eventually there are only small changes in particle concentrations and formation rates. This overshoot can be quite large and, in this example, reaches almost a factor of 3 for the maximum $J$ compared with its steady-state value.

Using the size distribution as a function of time for particle sizes equal to or larger than $d_{p 2}$ (not shown), as well as the growth rates $\mathrm{GR}_{m+1}$ (not shown) and the time derivative of the total number concentration of particles $\mathrm{d} N_{m+1} / \mathrm{d} t$, the size-corrected formation rate $J_{d p 1}$ can be derived by the method described in Sect. 2.3 (solid red line). The derived formation rate agrees closely with the accurate solution from the kinetic model (solid green line) for conditions close to steady state. However, when evaluating $J$ at $d_{p 1}$ from the formation rate at $d_{p 2}$ and time $t$, one needs to consider that the particles that appear at $d_{p 2}$ were passing the size $d_{p 1}$ at an earlier time $t^{\prime}$. This time can be approximated by

$t^{\prime}=t-\frac{d_{p 2}-d_{p 1}}{\mathrm{GR}_{m+1}(t)}$

if the time dependency of the GR is considered. Displaying the reconstructed formation rate $J\left(d_{p 1}\right)$ against the corrected time axis yields the dash-dotted red line, which shows a very similar time dependency to the accurate $J\left(d_{p 1}\right)$. The overestimation (difference between the red and green lines in Fig. 6) is due to the size dependency of the growth rate (see previous section). An accurate determination of $J\left(d_{p 1}\right)$ can only be obtained after the particles have formed at and above $d_{p 2}$.

\subsection{Formation rates as a function of the sulfuric monomer concentration}

\subsubsection{Kinetic limit}

In the preceding section, the universal method has only been tested for one sulfuric acid monomer concentration. Variation of the monomer production rate $P_{1}$ in Eq. (22) will result in different sulfuric acid concentrations. The resulting size distributions $(N)$, growth rates (GR), and rates of change of particle concentration $(\mathrm{d} N / \mathrm{d} t)$ as a function of particle size can be used to test the reconstruction method. Figure 7 shows the results for $8 \times 10^{5}$ to $2 \times 10^{7} \mathrm{~cm}^{-3}$ sulfuric acid concentration (lines denoting "kinetic limit"). The accurate solution for $d_{p 2}$ is shown by the solid blue line, while $J\left(d_{p 1}\right)$ is shown by the solid green line. Using a constant GR, corresponding to its value at $d_{p 2}$, the reconstruction method yields the results shown by the solid red line. For the high nucleation rates (above several hundred) the accuracy is quite good. For the lower formation rates, the required corrections are quite large because the growth between 1.4 and $2.9 \mathrm{~nm}$ is slow and therefore losses are high. The effect of the size-dependent growth rate therefore has a relatively large impact on the reconstructed NPF rates. The curved shape of the formation rates displayed against the sulfuric acid concentration on a $\log -\log$ plot is due to the fact that losses are much more relevant when particle growth is slow (see Ehrhart and Curtius, 2013).

In practice, GR will always be subject to measurement uncertainties. In order to test the sensitivity of the method, the constant GR was multiplied by both a factor of 1.5 and 0.9. The faster GR leads to an underestimation in the reconstructed $J\left(d_{p 1}\right)$, while the slower GR leads to an overestimation. The variation in the GR is indicated by the light-red bands in Fig. 7 . It can be seen that the reconstructed $J\left(d_{p 1}\right)$ is highly sensitive to GR, especially when the particle growth is slow. In this example, a GR underestimation of only $10 \%$ can lead to a substantial overestimation of $J\left(d_{p 1}\right)$ due to the exponential dependence on GR. Therefore, accurate growth rate measurements are essential to reliably reconstruct the particle formation rate at a smaller size.

In order to test the effect of self-coagulation, coagulation has only been taken into account to occur with particles at and above $d_{p 2}$ (dashed black lines). As long as the formation rate is close to $\sim 100 \mathrm{~cm}^{-3} \mathrm{~s}^{-1}$ at $d_{p 1}$, the effect of neglecting self-coagulation is quite small. For larger formation rates the deviation progressively increases because selfcoagulational loss becomes competitive and eventually dominant compared to wall loss, dilution, and coagulation with larger particles. However, these numbers are relevant for the CLOUD chamber experiment and are not necessarily applicable to other chambers with other wall loss and dilution characteristics. Performing the corrections twice - once by including self-coagulation and a second time by neglecting it - over a range of formation rates can help to find the for- 


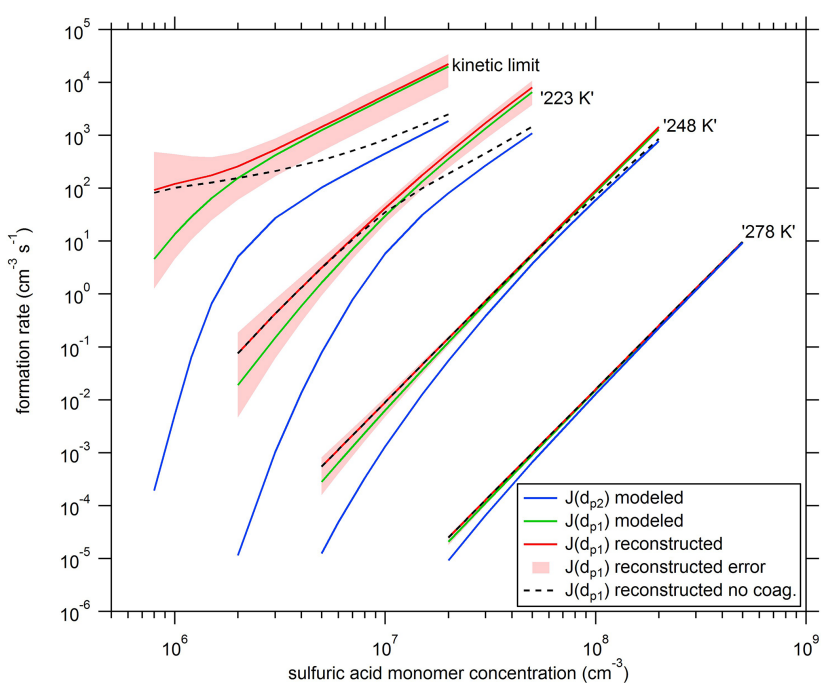

Figure 7. Formation rates as a function of the sulfuric acid monomer concentration. The solid blue curves show the formation rates at $d_{p 2}$ calculated from the model. The simulated formation rates $J\left(d_{p 1}\right)$ from the model are indicated by the green lines. The reconstructed formation rates at $d_{p 1}$ are shown by the red lines. Varying the constant GR by both a factor of 1.5 and 0.9 results in the error band shown in light red. Neglecting self-coagulation yields the dashed black line. A complete set of all curves is shown for four different scenarios (kinetic limit, "223 K", "248 K", and "278 K"). See text for details.

mation rate at which self-coagulation becomes important in other chambers. The advantage of neglecting coagulation is that the reconstruction is computationally much less demanding. One major difference between most experiments and the example calculations shown in Fig. 7 is that nucleation is generally not proceeding at the kinetic limit, even though this is the case for nucleation of sulfuric acid and dimethylamine (Kürten et al., 2014). In order to evaluate the method for NPF which is not proceeding at the kinetic limit, we have also simulated NPF with nonzero dimer and trimer evaporation rates.

\subsection{2 $223 \mathrm{~K}$}

The dimer evaporation rate has been set to $2.9 \mathrm{~s}^{-1}$ and the trimer evaporation rate to $0.024 \mathrm{~s}^{-1}$ (corresponding to conditions in the binary system at $223.15 \mathrm{~K}$ and $38 \% \mathrm{RH}$; see Hanson and Lovejoy, 2006). At these relatively low evaporation rates the effect of cluster-cluster collisions is still pronounced, which can be seen for the high sulfuric acid concentrations where a relatively large difference between $J\left(d_{p 2}\right)$ and $J\left(d_{p 1}\right)$ can be seen. This difference is due to the strong effect of self-coagulation, which leads to high loss rates. Although the GR is increasing with higher sulfuric acid concentration, self-coagulation increases as well because the cluster concentrations increase. Therefore, the two opposing effects cancel out, which leads to a rather constant factor between $J\left(d_{p 2}\right)$ and $J\left(d_{p 1}\right)$. The maximum deviation between the reconstructed and the accurate $J$ reaches a factor of 4 at the lowest sulfuric acid concentration of $2 \times 10^{6} \mathrm{~cm}^{-3}$. As the growth rate becomes higher and the corrections smaller with increasing sulfuric acid concentration, the effect of the size-dependent GR becomes less relevant and the accuracy increases.

\subsection{3 $248 \mathrm{~K}$}

Evaporation rates of respectively 181 and $3.1 \mathrm{~s}^{-1}$ were used for the dimer and the trimer (Hanson and Lovejoy, 2006). Because of these relatively high evaporation rates, particle formation and growth is dominated by collisions with monomers. The growth rates are quite high and selfcoagulation can be neglected for most conditions (monomer concentration below $\sim 1 \times 10^{8} \mathrm{~cm}^{-3}$ ); therefore the correction factors are lower than for the previous two conditions discussed. The maximum error due to the size-dependent GR is a factor of 2 at $5 \times 10^{6} \mathrm{~cm}^{-3}$ of sulfuric acid.

\subsection{4 $278 \mathrm{~K}$}

When evaporation rates of respectively 10060 and $360 \mathrm{~s}^{-1}$ are used for the dimer and the trimer, conditions can be simulated where monomer collisions are by far the dominant process for nucleation and growth due to very low cluster concentrations. In this case, quite high sulfuric acid monomer concentrations are required to yield substantial NPF. At these conditions the GRs are very high (up to $\sim 100 \mathrm{~nm} \mathrm{~h}^{-1}$ ) and self-coagulation is irrelevant. Therefore, the correction factor between $J\left(d_{p 2}\right)$ and $J\left(d_{p 1}\right)$ approaches a value of 1. Only at the low sulfuric acid monomer concentrations is a significant correction necessary.

\section{Conclusions}

The Kerminen and Kulmala (2002) method, and its refinements presented in subsequent publications (Lehtinen et al., 2007; Anttila et al., 2010; Korhonen et al., 2014), is widely used in atmospheric and chamber experiments to derive nucleation rates from experimentally measured formation rates at larger particle sizes. However, it was not designed to be applied to chamber nucleation experiments where selfcoagulation can be important.

We have therefore presented a new method that yields representative results in any general environment, provided certain quantities are known. The new method requires knowledge of the particle size spectrum above the detection threshold, the particle growth rate, and all loss processes as a function of particle size. With this information the size spectrum and the formation rate can be reconstructed in a stepwise process to a smaller size, where the nucleation rate is determined. The method can give accurate results and, furthermore, takes into account self-coagulation among newly formed particles, which can be an important effect, recog- 
nized previously by Anttila et al. (2010). Additionally, if the size-dependent growth rate is available from measurements, it can be readily incorporated during the reconstruction of the size distribution.

The proposed new method allows extrapolation of the particle formation rate measured at one threshold size $\left(d_{p 2}\right)$ to a second, smaller size $\left(d_{p 1}\right)$. In this way, a precise quantitative comparison can be made between formation rates measured simultaneously by several counters operating in the 1 to $3 \mathrm{~nm}$ threshold range and, where differences emerge, a deeper understanding of fundamental quantities such as cluster critical sizes, growth rates, and evaporation rates can be obtained.

One general issue with all methods that extrapolate formation rates towards smaller sizes arises from the uncertainty in the growth rate. In most cases no measurement of the GR will be available down to the very small size since the particle number concentrations are also not available (otherwise no extrapolation of the formation rate would be necessary). A small size dependency of the GR that is not taken into account can therefore lead to large uncertainty. In addition, the critical size of the nucleating particles is generally not known. Ideally, one would choose $d_{p 1}$ to correspond to the critical size. However, since this is not possible, a reasonable solution to this issue is to choose a size for $d_{p 1}$ which is safely at or above the critical size in order to avoid extending the size distribution into the subcritical size regime. For this reason the CLOUD experiment has reported particle formation rates at a size of $1.7 \mathrm{~nm}$ in mobility diameter rather than nucleation rates (Kirkby et al., 2011).

Further studies using the new method will focus on the effect of using larger size bins and its application to experimental data measured with condensation particle counters (CPCs) and scanning mobility particle sizer (SMPS) systems.

Acknowledgements. This research received funding from the EC Seventh Framework Programme (Marie Curie Initial Training Network MC-ITN "CLOUD-TRAIN" no. 316662) and the German Federal Ministry of Education and Research (project no. 01LK1222A). We thank Tinja Olenius and Rick Flagan for helpful discussions.

Edited by: H. Grothe

\section{References}

Almeida, J., Schobesberger, S., Kürten, A., Ortega, I. K., Kupiainen-Määttä, O., Praplan, A. P., Adamov, A., Amorim, A., Bianchi, F., Breitenlechner, M., David, A., Dommen, J., Donahue, N. M., Downard, A., Dunne, E. M., Duplissy, J., Ehrhart, S., Flagan, R. C., Franchin, A., Guida, R., Hakala, J., Hansel, A., Heinritzi, M., Henschel, H., Jokinen, T., Junninen, H., Kajos, M., Kangasluoma, J., Keskinen, H., Kupc, A., Kurtén, T., Kvashin, A. N., Laaksonen, A., Lehtipalo, K., Leiminger, M., Leppä, J., Loukonen, V., Makhmutov, V., Mathot, S., McGrath, M. J., Nieminen, T., Olenius, T., Onnela, A., Petäjä, T., Riccobono, F., Riipinen, I., Rissanen, M., Rondo, L., Ruuskanen, T., Santos, F. D., Sarnela, N., Schallhart, S., Schnitzhofer, R., Seinfeld, J. H., Simon, M., Sipilä, M., Stozhkov, Y., Stratmann, F., Tomé, A., Tröstl, J., Tsagkogeorgas, G., Vaattovaara, P., Viisanen, Y., Virtanen, A., Vrtala, A., Wagner, P. E., Weingartner, E., Wex, H., Williamson, C., Wimmer, D., Ye, P., Yli-Juuti, T., Carslaw, K. S., Kulmala, M., Curtius, J., Baltensperger, U., Worsnop, D. R., Vehkamäki, H., and Kirkby, J.: Molecular understanding of sulphuric acid-amine particle nucleation in the atmosphere, Nature, 502, 359-363, 2013.

Anttila, T., Kerminen, V.-M., and Lehtinen, K. E. J.: Parameterizing the formation rate of new particles: The effect of nuclei selfcoagulation, J. Aerosol Sci., 41, 621-636, 2010.

Chen, M., Titcombe, M., Jiang, J., Jen, C., Kuang, C., Fischer, M. L., Eisele, F. L., Siepmann, J. I., Hanson, D. R., Zhao, J., and McMurry, P. H.: Acid-base chemical reaction model for nucleation rates in the polluted atmospheric boundary layer, P. Natl. Acad. Sci. USA, 109, 18713-18718, doi:10.1073/pnas.1210285109, 2012.

Crump, J. G. and Seinfeld, J. H.: Turbulent deposition and gravitational sedimentation of an aerosol in a vessel of arbitrary shape, J. Aerosol Sci., 12, 405-415, 1981.

Ehrhart, S. and Curtius, J.: Influence of aerosol lifetime on the interpretation of nucleation experiments with respect to the first nucleation theorem, Atmos. Chem. Phys., 13, 11465-11471, doi:10.5194/acp-13-11465-2013, 2013.

Hanson, D. R. and Lovejoy, E. R.: Measurement of the thermodynamics of the hydrated dimer and trimer of sulfuric acid, J. Phys. Chem. A, 110, 9525-9528, 2006.

Heisler, S. L. and Friedlander, S. K.: Gas-to-particle conversion in photochemical smog: aerosol growth laws and mechanisms for organics, Atmos. Environ., 11, 157-168, 1977.

Hinds, W. C.: Aerosol Technology: Properties, Behavior, and Measurement of Airborne Particles, John Wiley \& Sons, New York, 150-154, 1999.

Iida, K., Stolzenburg, M. R., and McMurry, P. H.: Effect of working fluid on sub-2 $\mathrm{nm}$ particle detection with a laminar flow ultrafine condensation particle counter, Aerosol Sci. Technol., 43, 81-96, 2009.

Kangasluoma, J., Kuang, C., Wimmer, D., Rissanen, M. P., Lehtipalo, K., Ehn, M., Worsnop, D. R., Wang, J., Kulmala, M., and Petäjä, T.: Sub-3 nm particle size and composition dependent response of a nano-CPC battery, Atmos. Meas. Tech., 7, 689-700, doi:10.5194/amt-7-689-2014, 2014.

Kerminen, V. M. and Kulmala, M.: Analytical formulae connecting the "real" and the "apparent" nucleation rate and the nuclei number concentration for atmospheric nucleation events, J. Aerosol Sci., 33, 609-622, 2002. 
Kerminen, V.-M., Anttila, T., Lehtinen, K., and Kulmala, M.: Parameterization for atmospheric new-particle formation: application to a system involving sulfuric acid and condensable watersoluble organic vapors, Aerosol Sci. Technol., 38, 1001-1008, 2004.

Kirkby, J., Curtius, J., Almeida, J., Dunne, E., Duplissy, J., Ehrhart, S., Franchin, A., Gagné, S., Ickes, L., Kürten, A., Kupc, A., Metzger, A., Riccobono, F., Rondo, L., Schobesberger, S., Tsagkogeorgas, G., Wimmer, D., Amorim, A., Bianchi, F., Breitenlechner, M., David, A., Dommen, J., Downard, A., Ehn, M., Flagan, R.C., Haider, S., Hansel, A., Hauser, D., Jud, W., Junninen, H., Kreissl, F., Kvashin, A., Laaksonen, A., Lehtipalo, K., Lima, J., Lovejoy, E. R., Makhmutov, V., Mathot, S., Mikkilä, J., Minginette, P., Mogo, S., Nieminen, T., Onnela, A., Pereira, P., Petäjä, T., Schnitzhofer, R., Seinfeld, J. H., Sipilä, M., Stozhkov, Y., Stratmann, F., Tomé, A., Vanhanen, J., Viisanen, Y., Vrtala, A., Wagner, P. E., Walther, H., Weingartner, E., Wex, H., Winkler, P. M., Carslaw, K. S., Worsnop, D. R., Baltensperger, U., and Kulmala, M.: Role of sulphuric acid, ammonia and galactic cosmic rays in atmospheric aerosol nucleation, Nature, 476, 429-435, 2011.

Korhonen, H., Kerminen, V.-M., Kokkola, H., and Lehtinen, K. E. J.: Estimating atmospheric nucleation rates from size distribution measurements: Analytical equations for the case of size dependent growth rates, J. Aerosol Sci., 69, 13-20, 2014.

$\mathrm{Ku}, \mathrm{B} . \mathrm{K}$. and Fernandez de la Mora, J.: Relation between electrical mobility, mass, and size for nanodrops $1-6.5 \mathrm{~nm}$ in diameter in air, Aerosol Sci. Technol., 43, 241-249, 2009.

Kuang, C., Chen, M., McMurry, P. H., and Wang, J.: Modification of laminar flow ultrafine condensation particle counters for the enhanced detection of $1 \mathrm{~nm}$ condensation nuclei, Aerosol Sci. Technol., 46, 309-315, 2012a.

Kuang, C., Chen, M., Zhao, J., Smith, J., McMurry, P. H., and Wang, J.: Size and time-resolved growth rate measurements of 1 to $5 \mathrm{~nm}$ freshly formed atmospheric nuclei, Atmos. Chem. Phys., 12, 3573-3589, doi:10.5194/acp-12-3573-2012, 2012 b.

Kulmala, M., Vehkamäki, H., Petäjä, T., Dal Maso, M., Lauri, A., Kerminen, V.-M., Birmili, W., and McMurry, P. H.: Formation and growth rates of ultrafine atmospheric particles: a review of observations, J. Aerosol Sci., 35, 143-176, 2004.

Kulmala, M., Kontkanen, J., Junninen, H., Lehtipalo, K., Manninen, H. E., Nieminen, T., Petäjä, T., Sipilä, M., Schobesberger, S., Rantala, P., Franchin, A., Jokinen, T., Järvinen, E., Äijälä, M., Kangasluoma, J., Hakala, J., Aalto, P. P., Paasonen, P., Mikkilä, J., Vanhanen, J., Aalto, J., Hakola, H., Makkonen, U., Ruuskanen, T., Mauldin III, R. L., Duplissy, J., Vehkamäki, H., Bäck, J., Kortelainen, A., Riipinen, I., Kurtén, T., Johnston, M. V., Smith, J. N., Ehn, M., Mentel, T. F., Lehtinen, K. E. J., Laaksonen, A., Kerminen, V.-M., and Worsnop, D. R.: Direct observations of atmospheric aerosol nucleation, Science, 339, 943-946, 2013.

Kürten, A., Jokinen, T., Simon, M., Sipilä, M., Sarnela, N., Junninen, H., Adamov, A., Almeida, J., Amorim, A., Bianchi, F., Breitenlechner, M., Dommen, J., Donahue, N. M., Duplissy, J., Ehrhart, S., Flagan, R. C., Franchin, A., Hakala, J., Hansel, A., Heinritzi, M., Hutterli, M., Kangasluoma, J., Kirkby, J., Laaksonen, A., Lehtipalo, K., Leiminger, M., Makhmutov, V., Mathot, S., Onnela, A., Petäjä, T., Praplan, A. P., Riccobono, F., Rissanen, M. P., Rondo, L., Schobesberger, S., Seinfeld, J. H., Steiner, G., Tomé, A., Tröstl, J., Winkler, P. M., Williamson, C., Wimmer, D., Ye, P., Baltensperger, U., Carslaw, K. S., Kulmala, M.,
Worsnop, D. R., and Curtius, J.: Neutral molecular cluster formation of sulfuric acid-dimethylamine observed in real-time under atmospheric conditions, P. Natl. Acad. Sci. USA, 111, 1501915024, doi:10.1073/pnas.1404853111, 2014.

Landgrebe, J. D. and Pratsinis, S. E.: A discrete-sectional model for particulate production by gas-phase chemical reaction and aerosol coagulation in the free-molecular regime, J. Colloid Interf. Sci., 139, 63-86, 1990.

Lehtinen, K. E. J., dal Maso, M., Kulmala, M., and Kerminen, V.M.: Estimating nucleation rates from apparent particle formation rates and vice versa: Revised formulation of the KerminenKulmala equation, J. Aerosol Sci., 38, 988-994, 2007.

Lovejoy, E. R., Curtius, J., and Froyd, K. D.: Atmospheric ioninduced nucleation of sulfuric acid and water, J. Geophys. Res., 109, D08204, doi:10.1029/2003JD004460, 2004.

McMurry, P. H.: Photochemical Aerosol Formation from $\mathrm{SO}_{2}$ : A theoretical analysis of smog chamber data, J. Colloid Interf. Sci., 78, 513-527, 1980.

McMurry, P. H., Fink, M., Sakurai, H., Stolzenburg, M. R., Mauldin III, R. L., Smith, J., Eisele, F., Moore, K., Sjostedt, S., Tanner, D., Huey, L. G., Nowak, J. B., Edgerton, E., and Voisin, D.: A criterion for new particle formation in the sulfur-rich Atlanta atmosphere, J. Geophys. Res., 110, D22S02, doi:10.1029/2005JD005901, 2005.

Metzger, A., Verheggen, B., Dommen, J., Duplissy, J., Prevot, A. S. H., Weingartner, E., Riipinen, I., Kulmala, M., Spracklen, D. V., Carslaw, K. S., and Baltensperger, U.: Evidence for the role of organics in aerosol particle formation under atmospheric conditions, P. Natl. Acad. Sci. USA, 107, 6646-6651, 2010.

Nieminen, T., Lehtinen, K. E. J., and Kulmala, M.: Sub-10 nm particle growth by vapor condensation - effects of vapor molecule size and particle thermal speed, Atmos. Chem. Phys., 10, 9773 9779, doi:10.5194/acp-10-9773-2010, 2010.

Olenius, T., Riipinen, I., Lehtipalo, K., and Vehkamäki, H.: Growth rates of atmospheric molecular clusters based on appearance times and collision-evaporation fluxes: Growth by monomers, J. Aerosol Sci., 78, 55-70, 2014.

Riccobono, F., Schobesberger, S., Scott, C. E., Dommen, J., Ortega, I. K., Rondo, L., Almeida, J., Amorim, A., Bianchi, F., Breitenlechner, M., David, A., Downard, A., Dunne, E. M., Duplissy, J., Ehrhart, S., Flagan, R. C., Franchin, A., Hansel, A., Junninen, H., Kajos, M., Keskinen, H., Kupc, A., Kürten, A., Kvashin, A. N., Laaksonen, A., Lehtipalo, K., Makhmutov, V., Mathot, S., Nieminen, T., Onnela, A., Petäjä, T., Praplan, A. P., Santos, F. D., Schallhart, S., Seinfeld, J. H., Sipilä, M., Spracklen, D. V., Stozhkov, Y., Stratmann, F., Tomé, A., Tsagkogeorgas, G., Vaattovaara, P., Viisanen, Y., Vrtala, A., Wagner, P. E., Weingartner, E., Wex, H., Wimmer, D., Carslaw, K. S., Curtius, J., Donahue, N. M., Kirkby, J., Kulmala, M., Worsnop, D. R., and Baltensperger, U.: Oxidation products of biogenic emissions contribute to nucleation of atmospheric particles, Science, 344, 717 721, 2014.

Seinfeld, J. H. and Pandis, S. N.: Atmospheric chemistry and physics: from air pollution to climate change, 2nd Edn., John Wiley \& Sons, Inc., Hoboken, NJ, 595-610, 2006.

Sgro, L. A. and Fernández de la Mora, J.: A simple turbulent mixing $\mathrm{CNC}$ for charged particle detection down to $1.2 \mathrm{~nm}$, Aerosol Sci. Technol., 38, 1-11, 2004. 
Vanhanen, J., Mikkilä, J., Lehtipalo, K., Sipilä, M., Manninen, H. E., Siivola, E., Petäjä, T., and Kulmala, M.: Particle size magnifier for nano-CN detection, Aerosol Sci. Technol., 45, 533-542, 2011.

Weber, R. J., Marti, J. J., McMurry, P. H., Eisele, F. L., Tanner, D. J., and Jefferson, A.: Measurements of new particle formation and ultrafine particle growth rates at a clean continental site, J. Geophys. Res., 102, 4375-4385, 1997.
Wimmer, D., Lehtipalo, K., Franchin, A., Kangasluoma, J., Kreissl, F., Kürten, A., Kupc, A., Metzger, A., Mikkilä, J., Petäjä, T., Riccobono, F., Vanhanen, J., Kulmala, M., and Curtius, J.: Performance of diethylene glycol-based particle counters in the sub-3 nm size range, Atmos. Meas. Tech., 6, 1793-1804, doi:10.5194/amt-6-1793-2013, 2013. 